\title{
1 Interplay between the Mediterranean diet and C-reactive protein genetic polymorphisms towards inflammation in adolescents.
}

\author{
Aline B. Arouca ${ }^{\mathrm{a}^{*}}$, Aline Meirhaeghe ${ }^{\mathrm{b}}$, Jean Dallongeville ${ }^{\mathrm{b}}$, Luis A. Moreno ${ }^{\mathrm{c}, \mathrm{d}, \mathrm{e}}$, Gustavo Jacob
}

4 Lourenço ${ }^{\mathrm{f}}$, Ascensión Marcos ${ }^{\mathrm{g}}$, Inge Huybrechts ${ }^{\mathrm{a}, \mathrm{h}}$, Yannis Manios ${ }^{\mathrm{i}}$, Christina-Paulina Lambrinou,

5 Frederic Gottrand ${ }^{\mathrm{j}}$, Anthony Kafatos ${ }^{\mathrm{k}}$, Mathilde Kersting ${ }^{1}$, Michael Sjöström $^{\mathrm{m}}$, Kurt Widhalm ${ }^{\mathrm{n}}$, Marika

6 Ferrari $^{\mathrm{o}}$, Denes Molnár ${ }^{\mathrm{p}}$, Marcela González-Gross ${ }^{\mathrm{e}, \mathrm{q}}$, Maria Forsner ${ }^{\mathrm{r}}$, Stefaan De Henauw ${ }^{\mathrm{a}}$, Nathalie

Michels ${ }^{\mathrm{a}}$ : the HELENA study group.
8 a'Department of Public Health, Faculty of Medicine and Health Sciences, Ghent University, Belgium. (Stefaan.DeHenauw@UGent.be;
$9 \quad$ Nathalie.michels@ugent.be)
10 bUMR1167, RID-AGE, Risk Factors and Molecular Determinants of Aging-Related Diseases, Centre Hosp. Univ Lille, Institut Pasteur de Lille,
11 Université de Lille, Lille, France. (aline.meirhaeghe@pasteur-lille.fr; jean.dallongeville@ pasteur-lille.fr)
12 'GENUD: “Growth, Exercise, Nutrition and Development” Research Group, Facultad de Ciencias de la Salud, University of Zaragoza, Spain
13 Instituto Agroalimentario de Aragon (IA2), Instituto de Investigacion Sanitaria Aragon (IIS Aragon), Zaragoza, Spain
14 'Centro de Investigacion Biomedica en Red de Fisiopatología de la Obesidad y Nutricion (CIBERObn), Instituto de Salud Carlos III, Madrid, Spain
15 faboratory of Cancer Genetics, Faculty of Medical Sciences, University of Campinas, Campinas, São Paulo, Brazil. (gutolour@ @cm.unicamp.br)
16 gDepartment of Metabolism and Nutrition, Institute of Food Science and Technology and Nutrition, Madrid, Spain. ( $\underline{\operatorname{amarcos} @ i c t a n . c s i c . e s)}$
17 hInternational Agency for Research on Cancer, Lyon, France. (huybrechtsI@iarc.fr)

18 iDepartment of Nutrition and Dietetics, School of Health Science \& Education, Harokopio University, Athens, Greece. (helena@hua.gr;

19 cplambrinos@gmail.com)

20 jFaculty of Medicine, University Lille, Lille, France. (Frederic.GOTTRAND@CHRU-LILLE.FR)

21 kFaculty of Medicine, University of Crete, Crete, Greece. (kafatos@med.uoc.gr)

22 'Research Department of Child Nutrition, Pediatric University Clinic, Ruhr-University Bochum, Germany. (mathilde.kersting@ruhr-uni23 bochum.de)

$24{ }^{\mathrm{m}}$ Department of Biosciences, Unit for Preventive Nutrition, Karolinska Institutet, Huddinge, Sweden. (michael.sjostrom@ki.se)

25 nepartment of Pediatrics, Div. Nutrition and Metabolism; Med. Univ. Vienna, Austria. (kurt.widhalm@meduniwien.ac.at)

26 Research Centre for Food and Nutrition, Council for Agricultural Research and Economics (CREA-AN), Italy. (marika.ferrari@ crea.gov.it)

27 pDepartment of Pediatrics, Medical School, University of Pécs, Pécs, Hungary. (molnar.denes@pte.hu)

28 IImFine Research Group. Department of Health and Human Performance, Facultad de Ciencias de la Actividad Física y del Deporte-INEF,

29 Universidad Politécnica de Madrid, Madrid, Spain. (marcela.gonzalez.gross@upm.es)

30 rDepartment of Nursing, Umeå University, Sweden. (mfr@du.se)

31 *Corresponding author: aline.barbedoarouca@ugent.be 
32 4K3, Corneel Heymanslaan 10, 9000 Ghent, Belgium Tel: +32 93323679

\section{ABSTRACT}

35 Aim: From a nutrigenetics perspective, we aim to investigate the moderating role of the Mediterranean diet

36 and each of its subgroups in the association between $\mathrm{C}$-reactive protein $(C R P)$ gene polymorphisms and

37 CRP blood concentration in adolescents.

38 Methods: In 562 adolescents (13-17y) of the European HELENA study, data was available on circulating

39 CRP levels as inflammatory biomarker, three CRP gene SNPs (rs3093068, rs1204, rs1130864), food intake

40 determined by a self-administered computerized 24h-dietary recall for 2 days, and body composition. A 9-

41 point Mediterranean diet score and each food subgroup were tested as moderator via SNP*diet interaction.

42 Analyzes were adjusted for age, sex, puberty, adiposity and socioeconomic status.

43 Results: The minor allele frequencies of rs3093068 and rs1130864 SNPs (GG and TT, respectively) were

44 associated with higher CRP concentrations, while rs1205 (CT/TT) was associated with lower CRP

45 concentrations. There were significant interactions between rs3093068 and Mediterranean diet $(B=-0.1139$,

$46 p=0.011)$, or the fish food subgroup $(\mathrm{B}=-0.0090, p=0.022)$, so that those with the highest genetic CRP risk

47 underwent the highest CRP attenuation by a healthier diet. Although the effect of diet and SNP was

48 substantial, the explained variance by interaction was only $1 \%$.

49 Conclusion: Greater adherence to the Mediterranean diet and particularly its fish component was associated

50 with a lower CRP blood concentrations especially in those at highest genetic risk due to the rs3093068

51 SNP.

52

53 Key words: C-reactive protein, Single Nucleotide Polymorphism, Mediterranean diet, inflammation, 54 interaction, nutrigenomics. 


\section{Introduction}

57 Inflammation is a major health risk linked with worldwide mortality, as it is present in most non-

58 communicable chronic diseases. As an example, the onset and progression of atherosclerosis as a

59 pathological process towards cardiovascular diseases (CVD) is based on the establishment of an

60 inflammatory process [1]. Changes in inflammation can be assessed by measuring inflammatory biomarkers

61 in the blood; C-reactive protein (CRP) is herein one of the most studied biomarkers [2]. CRP is not only

62 associated with acute inflammation, but also with other metabolic abnormalities [3] in which minor CRP

63 elevation is considered a marker of low-grade inflammation [4]. Although data are limited, associations

64 between CRP and CVD risk factors in children and adolescents are similar to those reported in adults [5-

65 7]. CRP concentrations in childhood are increased in the settings of obesity and are strongly associated with

66 waist circumference, body mass index, and adiposity [5,8-10].

67 Genetics play an important role in CRP concentrations. Extensive evidence shows that some single

68 nucleotide polymorphisms (SNPs) within the CRP gene at the 1q21-q23 region of the proximal long arm

69 of chromosome 1 (Figure 1, adapted from Lee et al. [11]) affect the amount of CRP produced with a

70 heritability around $30 \%$ to $40 \%$ [6]. Individual SNPs in the CRP gene might explain up to $10 \%$ of the

71 variance in blood CRP levels, e.g. the three CRP SNPs rs3093068, rs1205 and rs1130864 are shown to be

72 important influencers of CRP levels [12-14]. Genetic variation in genes involved in the etiology of

73 inflammation may interact with environmental exposures such as diet, to modulate susceptibility to

74 inflammation [15]. 


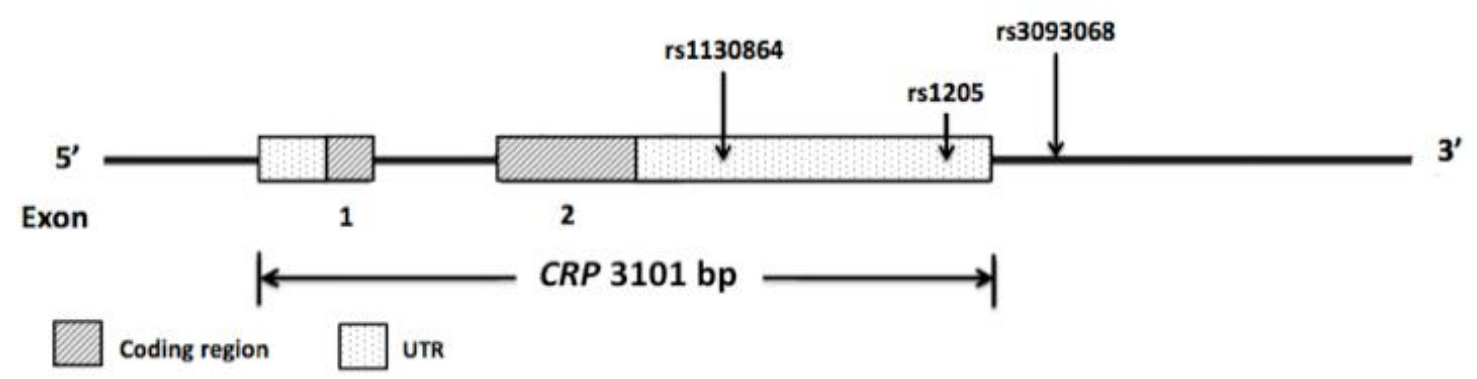

Adapted from Lee et al. [11]

78 Figure 1. The $C R P$ gene region (chromosome 1q21-q23) and the locations of the three SNPs tested in our 79 study. UTR, untranslated region.

80 Nutrigenetics is a raising research area which examines how the interactions between genetic 81 markers and phenotypic factors influence health [16]. Thus, the progression from a healthy phenotype to a

82 chronic disease phenotype occurs by changes in gene expression or enzyme and protein activities which

83 can be regulated by nutrients and bioactive dietary compounds [15]. Since certain foods can interact with

84 the transcription process of pro- and anti-inflammatory factor, a diet pattern might attenuate/exacerbate the 85 impact of genetic predisposition towards inflammation [17]. A healthy dietary intake, which is 86 characterized by the presence of essential nutrients, antioxidant vitamins, polyunsaturated (omega 3) and 87 monounsaturated fatty acids (as characterized by the Mediterranean diet) could attenuate low-grade 88 inflammation by improving postprandial dysmetabolism, and blunt the post-meal increase in glucose, 89 triglycerides, and oxidative stress [18]. In contrast, an energy-dense nutrient-low diet with non-essential fat 90 (e.g. trans-fat) and refined carbohydrates can immediately induce oxidative stress after a meal. This triggers 91 the increase of free radicals and atherogenic changes (including endothelial dysfunction, 92 hypercoagulability, sympathetic hyperactivity), contributing to low-grade inflammation and a future 93 cardiovascular event [19]. 
94 Indeed, Calder et al. [20] call for further research in this area, noting that the role of gene 95 polymorphisms in the effect of nutrients and dietary patterns on inflammation requires much greater

96 exploration. Testing this hypothesis in youth has the advantage of less confounding by existing chronic 97 disease or medication and can emphasize the importance of early prevention. Thus, this study aims (1) to 98 confirm whether genetic variation in three CRP SNPs (rs3093068, rs1204, rs1130864) is associated with 99 higher CRP blood concentrations already during adolescence, and (2) to explore whether a higher adherence 100 to the Mediterranean diet and each of its food subgroups could modulate this genetic susceptibility. After 101 all, the Mediterranean diet score has already been associated with lower inflammation in this young study 102 population [21].

103

\section{Methods}

\subsection{Study design and participants}

Data was derived from the HELENA Cross Sectional Study in 2006-2007 containing a sample of

107 adolescents from 10 European cities located in separated geographical points in Europe: Austria, Belgium,

108 France, Germany, Greece (two cities), Hungary, Italy, Spain and Sweden. The selected towns were 109 equivalent and comparable between countries. Their size was sufficiently large to ensure a diverse 110 population [22]. The main objective of the HELENA study was to obtain comparable data of a large sample

111 of European adolescents on nutrition and health related parameters by a standardized procedure. The study 112 was performed following the ethical guidelines of the Declaration of Helsinki 1961 (revision of Edinburgh 113 2000), the Good Clinical Practice, and the legislation about clinical research in humans in each of the 114 participating countries. All participants and their parents signed an informed consent.

115 The total HELENA sample was 3,528 adolescents in which a one-third subset $(\mathrm{n}=1,155)$ was 116 selected for blood sampling. A-priori it was decided based on sample size calculation that only around 1000

117 participants were needed for immunological parameters and school classes were thus randomly chosen for 118 blood collection. In the present study, 987 adolescents had data on both CRP blood concentration and CRP 119 SNPs (rs3093068, rs1205, and rs1130864). Specific inclusion criteria such as data availability from the 24- 
120 h dietary recall, anthropometry (measures on skinfold thickness, waist circumference), and data on 121 socioeconomic status, were defined in the present study for the interaction analyses, resulting in 562

122 adolescents (263 male, 299 female), aged 13.0-16.99 years (Figure 2). This further selection from 987 to

123562 adolescents did not introduce a significant difference in CRP levels or genotypes ( $p$ between 0.107 and

124 0.947).
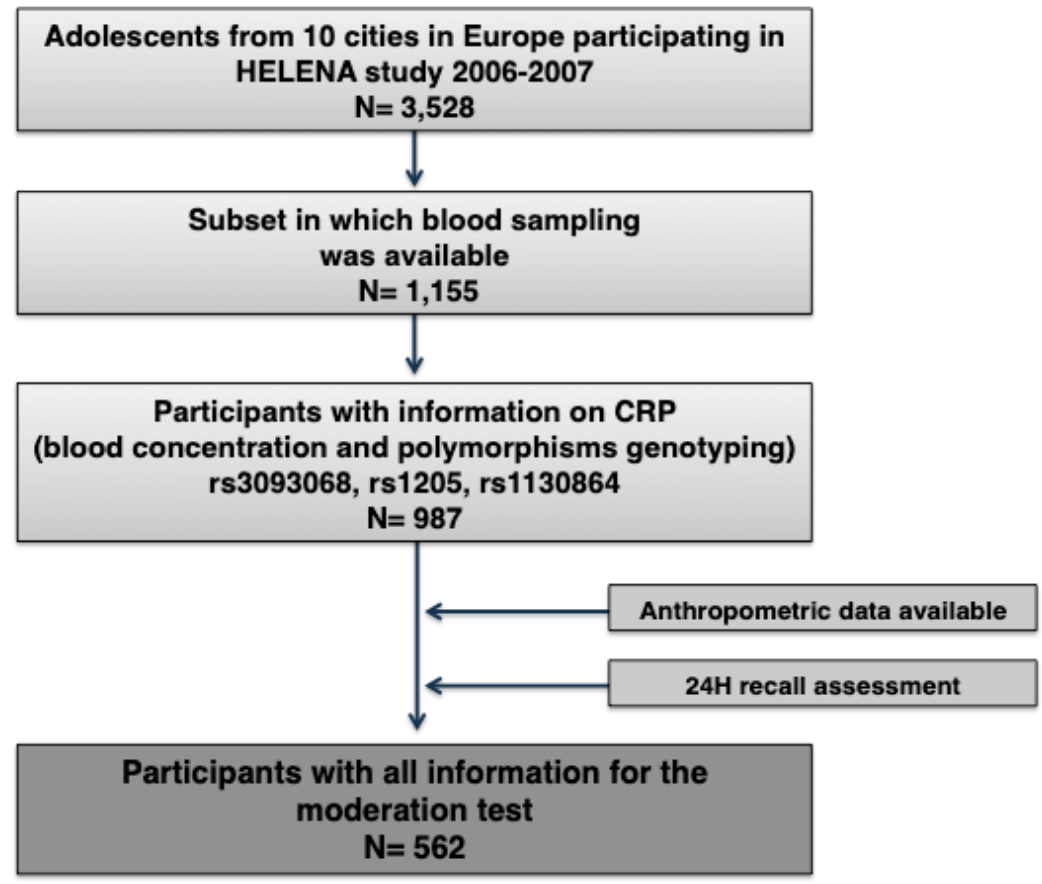

126 Figure 2. Sampling procedure scheme.

\subsection{Dietary intake assessment}

Eating habits were determined from a self-administered computerized validated 24-h dietary recall

129 called the HELENA-Dietary Assessment Tool (HELENA-DIAT) [23,24], a tool validated in Flemish

130 adolescents. Participants completed the HELENA-DIAT on two non-consecutive days within a time span

131 of 2 weeks. Based on these data, the Mediterranean diet score was calculated by a balance of low (score 0

132 to 4) versus high (score 5 to 9) adherence to the traditional Mediterranean diet.

\subsubsection{Mediterranean diet score}


135 saturated fatty acids, legumes, fruits and nuts, vegetables, meat, cereals, alcohol, dairy and fish. A scale 136 indicating the degree of adhesion to the traditional Mediterranean diet was constructed [25], and later

137 revised to include fish intake [26]. The adherence to the traditional Mediterranean diet was assessed by a

138 score ranging from 0 to 9 , with higher scores indicating greater adherence [27]. In this study, six

139 components in the Mediterranean diet score were considered as positive: 1) high ratio of monounsaturated

140 to saturated dietary lipids (mainly olive oil), 2) high consumption of vegetables, 3) high consumption of

141 fruits and nuts, 4) high consumption of fish, 5) high consumption of cereals, and 6) high consumption of

142 pulses; while three components were considered as negative: 7) high consumption of meat and meat

143 products, 8) high consumption of milk and dairy products, and 9) any consumption of alcohol. The alcohol

144 consumption (from alcoholic beverages) is presented as grams per day, for 25 consuming boys (from 263

145 subjects) and 66 consuming girls (from 299 subjects), and was considered negative because of our focus on

146 an adolescent population.

\section{$147 \quad$ 2.2.2 Mediterranean diet food-subgroups’ description}

The Vegetables food-subgroup consists of all types of vegetables excluding potatoes. The Fruits \&

149 Nuts food-subgroup consists of all types of fruits and nuts (including nut- and seed spreads). The Pulses

150 food-subgroup includes peas, beans, lentils, chickpeas, and fava beans (fresh peas and sweet corn were

151 excluded). The Cereal \& Roots food-subgroup is comprised by starch roots, potatoes and cereals such as

152 rice and wheat. The Monounsaturated/Saturated fat ratio food-subgroup is a ratio of mono-unsaturated fats

153 versus saturated fats. The Dairy food-subgroup is composed by all dairy products, such as: cow milk,

154 yoghurt, cheese, sour cream, and other milk products. The Fish food-subgroup is composed by all types of

155 fish and fish products. The Meat food-subgroup is composed by all types of meat, such as: chicken, pork,

156 cow, turkey, and veal meat and processed meat (such as ham, sausage, salami and mortadella).

$157 \quad 2.3$ Adiposity assessment 
To measure central adiposity, waist circumference was used. To reflect overall adiposity, the sum of 159 six skinfolds was used: skinfold thickness was measured to the nearest $0.2 \mathrm{~mm}$ in triplicate on the left side 160 at biceps, triceps, subscapular, suprailiac, thigh, and medial calf with a Holtain Caliper (Crymmych, UK).

161 Fieldworkers followed a central training and followed the same manual. Intraobserver reliability was above $16295 \%$, while interobserver reliability was above 90\% [28]. Body Mass Index (BMI) was categorized 163 following the IOTF cut-offs [29]. The anthropometric methods in the HELENA study have been reported 164 in detail elsewhere [28,30].

\subsection{Biochemical analyzes}

\subsubsection{Blood sample collection and CRP assessment}

In a randomly selected one-third subset of the total HELENA study population, blood samples were

168 collected in overnight fasting state. We used a standardized methodology for blood collection, transport

169 and analysis by a certified laboratory [31]. The quality control was within recommended levels and 170 transport had no influence. Detection limits (sensitivity) for serum CRP by immunoturbidimetry (AU2700

171 biochemistry analyzer, Olympus, Watford, UK) were $0.007 \mathrm{mg} / \mathrm{L}$ and $0.05 \mathrm{pg} / \mathrm{mL}$ with intra-assay CV of $1721.9 \%$. Nobody took nonsteroidal anti-inflammatory drugs or had an active inflammatory disease. None of 173 the girls had Polycystic Ovarian Syndrome or was in the menstrual period. Six individuals (only male) 174 presented CRP levels higher than $10 \mathrm{mg} / \mathrm{L}$. Only $8.4 \%$ smoked regularly.

\subsubsection{Single Nucleotide Polymorphisms genotyping}

176 Blood for DNA extraction was collected in EDTA K3 tubes and stored at IEL Bonn, and sent to 177 the Laboratoire d'Analyse Génomique Centre de Ressources Biologiques (LAG-CRB) - BB-0033-

17800071 Institut Pasteur de Lille, F-59000 Lille, France. DNA was extracted from white blood cells with the

179 Puregene kit (QIAGEN, Courtaboeuf, France) and stored at $-20^{\circ} \mathrm{C}$. Although several SNPs were genotyped 180 in this study, only three of these SNPs were located on the CRP gene: the rs3093068, rs1205, and 
181 rs1130864. The genotyping was done by an Illumina system (Illumina, Inc, San Diego, California) using

182 the Golden- Gate technology (GoldenGate Software, Inc, San Francisco, California). The genotyping 183 success rate was $100 \%$ for rs3093068 and rs1205, and was $99.9 \%$ for rs 1130864 . The genotype distributions

184 respected the Hardy-Weinberg equilibrium (HWE) for the $C R P \operatorname{rs} 3093068\left(\chi^{2}=1.15, p=0.28\right), \operatorname{rs} 1205\left(\chi^{2}=\right.$ $1851.08, p=0.30)$ and rs1130864 $\left(\chi^{2}=0.77, p=0.38\right)$ SNPs. Genotypes were coded as follows: CC, homozygous 186 major alleles; CG, heterozygotes; and GG, homozygous minor alleles for rs3093068, while CC; CT; and

187 TT, respectively, for rs1205 and rs1130864. The values for the linkage disequilibrium between the three 188 investigated SNPs was above 95 for D' and was between 3 and 20 for $\mathrm{r}^{2}$.

\subsection{Socioeconomic status (SES) and parental education}

As indicator of material affluence, the family affluence scale ranging from 0 (low) to 8 (high) was

192 based on family car ownership, having an own bedroom, Internet availability and computer ownership [30].

193 In addition, parental educational level was categorized into three score groups: primary education and lower

194 secondary education (score 1), higher secondary education (score 2), and tertiary education (score 3). A 195 detailed description of SES and parental education level can be found elsewhere [23].

$196 \quad 2.6$ Data analyses

197 Statistics were performed using SPSS (IBM SPSS Statistics, version 24.0) and moderation effects

198 were obtained by testing interactions $[32,33]$. The statistical significance was set at two-sided $p<0.05$. As

199 CRP levels were not normally distributed, the Mann-Whitney test was performed in comparing CRP levels

200 between groups and CRP was log-transformed for the regression analyses. The moderation effect of the 201 diet in the SNPs-CRP relation was tested by adding the interaction term "diet*SNP". In the case of a 202 significant interaction, the SNP relation was tested for 3 representative groups: those at the mean, at 1 SD 203 below the mean and 1 SD above the mean of the dietary index. Six individuals had high CRP levels (>10 $204 \mathrm{mg} / \mathrm{L}$ ) but were not excluded from the analyzes as it did not change any result. 


\section{Results}

\section{$207 \quad 3.1$ Characteristics of the study sample}

208 The participants' characteristics are shown in Table 1. Based on BMI, $13.7 \%$ of the adolescents (38

209 male; 39 female) were overweight, while 4.6\% (17 male; 9 female) were obese.

210

211 Table 1. Characteristics of the study sample.

$\mathrm{N}=562$ (263 males; 299 females)

\section{Median [P25; P75] or \%}

C-reactive protein $(\mathrm{mg} / \mathrm{L})$

$$
0.37[0.16-0.88]
$$

\begin{tabular}{lcc}
\hline & Mean & Std. Deviation \\
\cline { 2 - 3 } Age & & 14.81 \\
\hline
\end{tabular}

\section{Socio-economic characteristics}

High socioeconomic status $(\%)^{\mathrm{a}}$

$80.07 \%$

High paternal education $(\%)^{\mathrm{b}}$

$65.30 \%$

High maternal education $(\%)^{\mathrm{b}}$

$69.39 \%$

\section{Anthropometric characteristics}

Skinfold thickness (sum) (mm)

Body Mass Index (z-score)

Waist circumference $(\mathrm{cm})$
88.64

0.37

72.10

\section{Diet-related characteristics}

\footnotetext{
Vegetables (g/day)

Fruits and Nuts (g/day)
}

Mediterranean diet score $(0-9)$

4.15

1.45

93.96

129.38

10.56

Pulses (g/day)
39.75

1.08

8.25
129.38

56.61

100.05

34.09 


$\begin{array}{lcc}\text { Cereal and roots (g/day) } & 297.46 & 99.95 \\ \text { Monounsaturated/Saturated fat (ratio) } & 0.89 & 0.17 \\ \text { Dairy (g/day) } & 220.35 & 39.49 \\ \text { Fish (g/day) } & 18.48 & 19.42 \\ \text { Meat (g/day) } & 147.44 & 78.02 \\ \text { Alcohol consumption (g/d) } & 0.89 & 2.34 \\ \text { Energy (kcal) } & 2199.76 & 795.18 \\ \text { Total carbohydrate (g/day) } & 240.78 & 154.98 \\ \text { Total protein (g/day) } & 87.95 & 31.78 \\ \text { Total fat (g/day) } & 81.64 & 34.36 \\ \text { Cholesterol (mg/day) } & 248.81 & 16.16 \\ \text { Saturated fat (g/day) } & 44.94 & 18.22 \\ \text { Monounsaturated fat (g/day) } & 44.38 & 17.57 \\ \text { Polyunsaturated fat (g/day) } & 40.18 & 18.66 \\ \text { Eicosapentaenoic acid (mg/day) } & 17.11 & 2.87 \\ \text { Docosahexaenoic acid (mg/day) } & 20.89 & 2.99 \\ \text { Total fiber (g/day) } & 12.41 & 1.60\end{array}$

Data displayed for 562 adolescents (263 males; 299 females) who had all information for the moderation test: data on 24-h dietary recall, anthropometry, and SES. Values are presented as median or mean and standard deviation for continuous variables, and as \% (Chi square) for categorical variables. P25, 25th percentile; $\mathrm{P} 75$, 75th percentile; SD, standard deviation.

a Socioeconomic status was based on the family affluence scale (family car ownership, having an own bedroom, internet availability and computer ownership); a sum score from 4 to 8 represents medium to high socio-economic status.

b High parental education represents higher secondary education and tertiary education. Some variables were log-transformed as they were not normal distributed but backtransformed numbers are shown: saturated, monounsaturated and polyunsaturated fat; eicosapentaenoic and docosahexaenoic acid; total fiber.

\section{$213 \quad 3.2$ Associations between the allele frequencies of the investigated $C R P$ genotypes (rs3093068, rs1205}

214 and rs1130864) and CRP blood concentrations. 
217 SNPs were associated with higher CRP concentrations $(+0.15 \mathrm{mg} / \mathrm{L}, p=0.040$; and $+0.09 \mathrm{mg} / \mathrm{L}, p=0.004$,

218 respectively). In contrast, the minor allele of rs1205 was associated with lower CRP concentrations (-0.07

$219 \mathrm{mg} / \mathrm{L}, p=0.012)$.

220 Table 2. Associations between the allele frequencies of the investigated $C R P$ genotypes (rs3093068, rs1205

221 and rs1130864) and CRP blood concentrations in the HELENA study.

CRP SNPs

\begin{tabular}{|c|c|c|c|c|c|}
\hline & $\mathbf{N}$ & Median & Min-Max & Mean rank & $P$ value \\
\hline \multicolumn{6}{|l|}{ rs3093068 } \\
\hline $\mathrm{CC}$ & 834 & 0.37 & $0.01-75.99$ & 280.59 & \multirow[b]{2}{*}{0.042} \\
\hline $\mathrm{CG}$ or $\mathrm{GG}$ & 153 & 0.54 & $0.01-66.26$ & 286.73 & \\
\hline $\mathrm{C}$ allele & 1813 & 0.39 & $0.01-75.99$ & 561.92 & \multirow{2}{*}{0.043} \\
\hline G allele & 161 & 0.54 & $0.01-66.26$ & 569.52 & \\
\hline \multicolumn{6}{|l|}{ rs1205 } \\
\hline $\mathrm{CC}$ & 468 & 0.43 & $0.01-75.99$ & 512.85 & \multirow{2}{*}{0.049} \\
\hline $\mathrm{CT}$ or $\mathrm{TT}$ & 519 & 0.36 & $0.01-25.10$ & 477.00 & \\
\hline $\mathrm{C}$ allele & 1370 & 0.42 & $0.01-75.99$ & 1008.91 & \multirow{2}{*}{0.012} \\
\hline $\mathrm{T}$ allele & 604 & 0.35 & $0.01-25.10$ & 938.94 & \\
\hline \multicolumn{6}{|l|}{ rs1130864 } \\
\hline $\mathrm{CC}$ & 458 & 0.34 & $0.01-66.26$ & 467.60 & \multirow{2}{*}{0.008} \\
\hline $\mathrm{CT}$ or $\mathrm{TT}$ & 528 & 0.44 & $0.01-75.99$ & 515.97 & \\
\hline $\mathrm{C}$ allele & 1350 & 0.37 & $0.01-75.99$ & 961.60 & \multirow[t]{2}{*}{0.004} \\
\hline $\mathrm{T}$ allele & 622 & 0.46 & $0.01-75.99$ & 1040.53 & \\
\hline
\end{tabular}


229 3.3 Moderating effect of diet on the relation between $C R P$ gene SNPs (rs3093068, rs1205, rs1130864) 230 and CRP blood concentrations

Table 3 presents the interaction effects of diet on the relation between CRP concentrations and $C R P$

232 SNPs (rs3093068, rs1205, rs1130864, homozygous major allele carriers versus other genotypes) in 562

233 adolescents from the HELENA study. Significant interaction was detected for rs3093068 using the

234 Mediterranean diet score and the fish food subgroup $(\mathrm{B}=-0.1139, p=0.0110 ; \mathrm{B}=-0.0090, p=0.0220$;

235 respectively). As can be seen in Figure 3, higher adherence to the Mediterranean diet (panel A) or higher

236 fish intake (panel B) was associated with a slight attenuation of the CRP levels in those adolescents carrying

237 the genetic risk of rs3093068 SNP (G allele carriers). 
238 Table 3. Moderating effects of diet on the relation between CRP gene SNPs (rs3093068, rs1205, rs1130864) and CRP blood concentrations in 562 239 adolescents from the HELENA study.

\section{Food groups}

Mediterranean diet score

\begin{tabular}{l}
\hline Vegetables \\
\hline Fruits \& Nuts \\
\hline Pulses
\end{tabular}

\section{Cereal \& Roots \\ Monouns./Saturated fat ratio}

Dairy

\section{Fish}

Meat

Alcohol rs3093068

(CC vs CG+GG)

\begin{tabular}{ccc}
\hline B & $\mathbf{9 5 \%}$ CI & $\boldsymbol{p}$ \\
-0.1139 & $-0.2016--0.0262$ & $\mathbf{0 . 0 1}$
\end{tabular}

\begin{tabular}{ccc}
\hline-0.0014 & $-0.0033-0.0004$ & 0.13 \\
\hline-0.0004 & $-0.0015-0.0008$ & 0.53 \\
& & \\
\hline-0.0010 & $-0.0056-0.0036$ & 0.67 \\
& & \\
\hline
\end{tabular}

\begin{tabular}{lll}
\hline-0.0002 & $-0.0014-0.0011$ & 0.79
\end{tabular}

\begin{tabular}{lll}
\hline-0.4483 & $-1.1845-0.2879$ & 0.23 \\
& & \\
\hline 0.0002 & $-0.0005-0.0008$ & 0.61
\end{tabular}

\begin{tabular}{lll}
\hline-0.0090 & $-0.0166--0.0013$ & $\mathbf{0 . 0 2}$
\end{tabular}

\begin{tabular}{lll}
\hline 0.0002 & $-0.0015-0.0020$ & 0.79 \\
& & \\
\hline-0.0090 & $-0.0600-0.0420$ & 0.72
\end{tabular}

rs1205

(CC vs CT+TT)

\begin{tabular}{ccc}
\hline B & 95\% CI & $\boldsymbol{p}$ \\
-0.0077 & $-0.0699-0.0545$ & 0.80 \\
& & \\
\hline $1.21 \times 10^{-5}$ & $-0.0016-0.0016$ & 0.98 \\
& & \\
\hline 0.0004 & $-0.0006-0.0013$ & 0.44 \\
\hline-0.0016 & $-0.0043-0.0011$ & 0.24 \\
\hline-0.0006 & $-0.0015-0.0003$ & 0.19 \\
\hline 0.3199 & $-0.2071-0.8469$ & 0.23 \\
\hline-0.0003 & $-0.0007-0.0001$ & 0.19 \\
\hline 0.0013 & $-0.0034-0.0061$ & 0.58 \\
\hline 0.0001 & $-0.0011-0.0012$ & 0.92 \\
\hline-0.0118 & $-0.0580-0.0344$ & 0.61 \\
\hline
\end{tabular}

rs1130864

(CC vs CT+TT)

\begin{tabular}{ccc}
\hline $\mathbf{B}$ & $\mathbf{9 5 \%} \mathbf{C I}$ & $\boldsymbol{p}$ \\
0.0245 & $-0.0374-0.0864$ & 0.43 \\
& & 0.82 \\
\hline-0.0002 & $-0.0018-0.0014$ & 0.67 \\
\hline 0.0002 & $-0.0007-0.0011$ & 0.95 \\
\hline-0.0001 & $-0.0030-0.0028$ & 0.82 \\
\hline 0.0001 & $-0.0008-0.0010$ \\
\hline 0.0521 & $-0.4811-0.5853$ & 0.84 \\
\hline-0.0001 & $-0.0005-0.0003$ & 0.55 \\
\hline-0.0015 & $-0.0062-0.0032$ & 0.53 \\
\hline-0.0001 & $-0.0012-0.0011$ & 0.90 \\
\hline 0.0059 & $-0.0417-0.0534$ & 0.80 \\
\hline
\end{tabular}

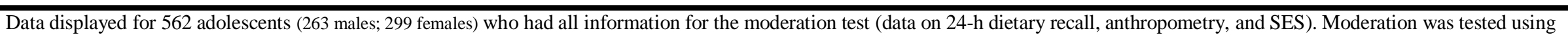

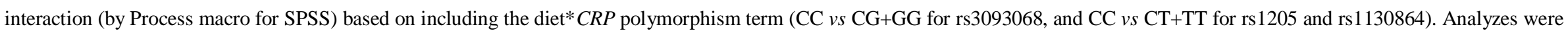

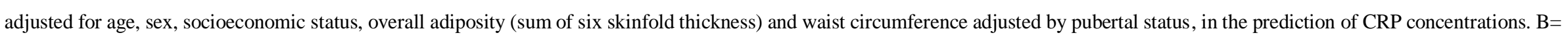
unstandardized coefficient. Bold: statistical significance when $p<0.05$. 
(A) Moderating effect of the adherence to the Mediterranean diet on CRP blood concentrations among adolescents with and without the CC genotype of rs3093068.

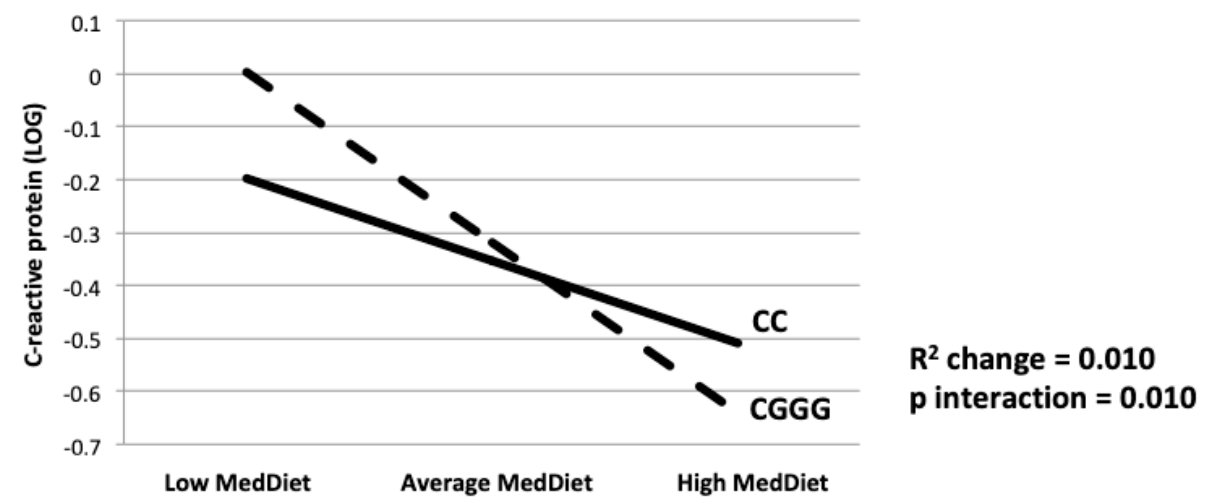

C-reactive protein level log transformed. Black straight line: CC, wild homozygous; black dotted line: CG, heterozygous, and GG, variant homozygous of the rs3093068 SNP. Analyzes were adjusted for age, sex, socioeconomic status, overall and central adiposity (skinfold thickness and waist circumference, respectively). In the case of a significant moderation, the CRP-SNP relation was tested for 3 representative groups: those at the mean (average adherence), at 1 SD below the mean (lower adherence), and 1 SD above the mean (higher adherence) of the dietary index.

(B) Moderating effect of the fish subgroup intake on CRP blood concentrations among adolescents with and without the CC genotype of rs3093068.

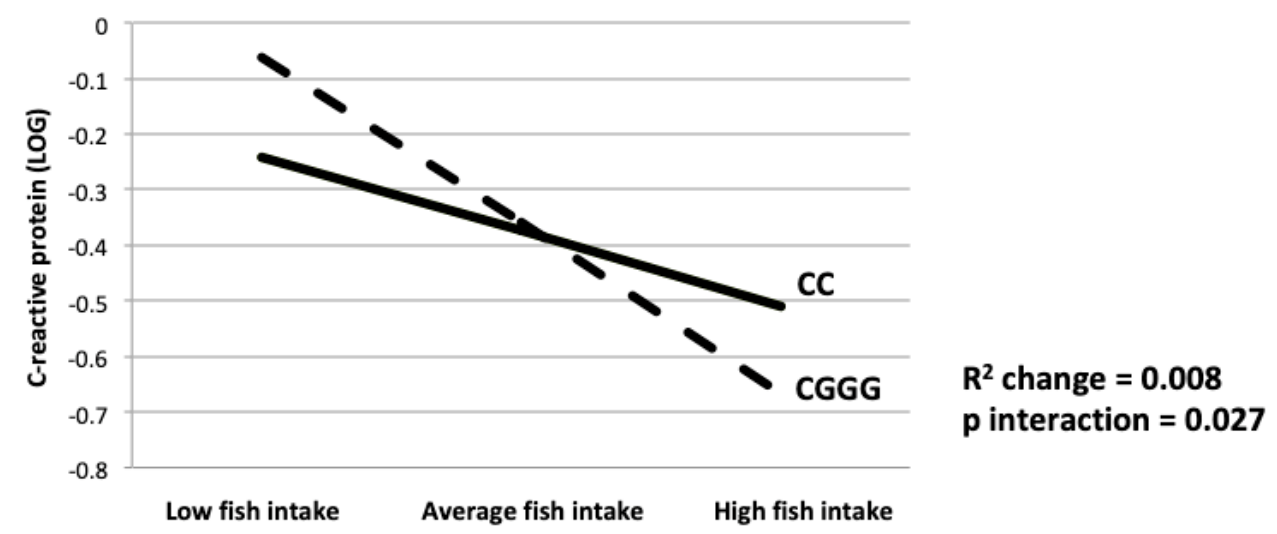

C-reactive protein level log transformed. Black straight line: CC, wild homozygous; black dotted line: CG, heterozygous, and GG, variant homozygous of the rs3093068 SNP. Analyzes were adjusted for age, sex, socioeconomic status, overall and central adiposity (skinfold 
thickness and waist circumference, respectively). In the case of a significant moderation, the CRP-SNP relation was tested for 3 representative groups: those at the mean (average fish intake), at 1 SD below the mean (lower fish intake), and 1 SD above the mean (higher fish intake).

\section{Discussion}

To our knowledge, this is the first study that investigates the capacity of the Mediterranean diet and its

248 food sub-groups in influencing inflammatory status (here measured with CRP concentrations) according to some selected CRP gene polymorphisms in European adolescents. The cross-sectional results showed that a higher Mediterranean diet adherence and fish intake seemed to attenuate a genetic risk (rs3093068) towards CRP-related inflammation. This was not the case for the two other SNPs.

Our first observation was that the genetic variants in the CRP gene were substantially associated with CRP concentrations, in agreement with other studies [34]. The minor G or T alleles of rs3093068 and rs 1130864 were significantly associated with higher CRP concentrations as demonstrated by some studies $[13,14]$ while minor allele T carriers of rs1205 associated with lower levels of CRP [11].

Second, the interaction between diet and SNPs regarding CRP blood concentrations was tested. Although slight, a higher Mediterranean diet adherence and fish intake attenuated the CRP blood concentrations for homozygous major alleles carriers (CC) but even more pronounced in heterozygotes and homozygous minor alleles carriers (CG/GG) that are genetically at higher inflammatory risk. The effect was small as the interaction term only explained around $1 \%$ additional variance in CRP concentrations. The CRP attenuation in the high-risk genetic group was double the size (around $0.6 \mathrm{mg} / \mathrm{L}$ ) compared to the attenuation in the low-risk genetic group (around $0.3 \mathrm{mg} / \mathrm{L}$ ).

This is in the expected direction as the Mediterranean diet has been associated with better metabolic and 264 inflammatory profiles in some clinical trials [35,36], and fish has anti-inflammatory potential [37]. Indeed, studies 265 show that a lower adherence to the Mediterranean diet leads to a worse profile of inflammatory markers in adults 266 and adolescents [38], while greater adherence brings significant improvements in lipid profile [39] and reduction 267 in risk of developing type 2 diabetes [40]. This can be explained mainly by specific food groups present in the 
268 Mediterranean diet such as vegetables, fruits, nuts, legumes, fish, and a high proportion of monounsaturated fat, 269 which all provide essential nutrients, antioxidant vitamins and minerals capable of interacting with genes [41]. As an example, green dark vegetables are rich in folate, which is crucial for DNA methylation status and thus epigenetics [42]. Regarding the fish subgroup, several of its nutrients might contribute to lower CRP: mainly long chain polyunsaturated fatty acids omega-3, but it also contains protein, iron, zinc, iodine, vitamin B12, vitamin A 273 (especially oily fish), and vitamin D [43]. In fact, these essential nutrients may lead to a better cardiovascular 274 health, hence the calls for higher fish consumption [44]. Using an overall dietary pattern score like our Mediterranean diet score has the advantage of considering the potential synergy between multiple dietary factors. Several studies have shown genotype-nutrient interactions, including interactions with SNPs that affect inflammation [15,45]. Nutrients, micronutrients and phytochemicals found in food are the most influential environmental stimuli that govern the expression of genetic information. From a preventive perspective, genetic predisposition conferring health disadvantages like inflammation, could possibly be attenuated with diet modifications [46]. A fish oil supplementation for 12 weeks in healthy young men showed a decrease in TNF- $\alpha$ production depending on the genotype [47]. A cross-sectional study in 2,010 South African adults also tested such SNP*diet interaction using $12 C R P$-related SNPs [48]. A high ratio of omega-6 to omega-3 intake was associated with higher CRP concentrations in those carrying the major alleles at SNPs rs3093058 and rs3093062, compared to those carrying the minor allele [48]. Yet, increased cholesterol intake led to increased CRP levels in those individuals carrying the minor allele in rs3093058 or rs3093062, while those homozygous for the major allele were more protected and were thus "low responders" to cholesterol intake [48]. These cholesterol and omega interactions were not found for the three SNPs we considered [48]. For none of the 12 SNPs, interactions with the diet quality score, fruits and vegetables, pulses/nuts/seeds, carbohydrates, added sugar, total fat, monounsaturated/saturated fat ratio, alcohol, fiber, vitamin C, vitamin E, zinc or magnesium were significant [48]. Interactions were thus SNP and dietary factor specific. Although there is absolutely no consensus, our findings factor. 
For the first time, we have shown that a Mediterranean diet could attenuate inflammation by gene-diet

296 interaction in adolescents. A first strength is the multi-country design of this study. As this investigation involved 297 the participation of adolescents from 10 European cities, this created the advantage of using a large database with 298 relevant information on the diversity of dietary intake e.g. both Mediterranean and non-Mediterranean countries. 299 Herein, we focus on an understudied population i.e. adolescents in the perspective of early treatment and 300 prevention. A second asset is that we tested three SNPs and a holistic view of using an overall dietary index with 301 sub-analyses on separate food groups allowing overall and specific public health recommendations. The analyses 302 303 were adjusted for adiposity as an important source of chronic inflammation: the waist circumference was used to reflect central adiposity, while we applied the sum of six skinfolds for overall adiposity, since it is considered as a better predictor of body fat than BMI [49].

The present study has some limitations. First, a self-administered computerized 24-h dietary recall relies on the respondents' memory and their capabilities to interpret those questions on frequency and quantity of consumption in the last 24 hours. Despite the assessment on two non-consecutive days, this may not reflect their eating habits on weekends or holidays. Second, the cross-sectional nature of our study does not permit causality statements. Third, a single measurement of CRP does not imply that there has been long-term inflammation as 310 CRP is a sensitive biomarker with high variability and can be influenced by interacting with e.g. metabolism. 311 Fourth, assessing SNPs of only one inflammatory parameter (CRP) is not enough to understand the complex 312 interactions that occur in inflammation, since inflammatory status is characterized by the production of a wide 313 range of mediators working in a complex network. Fifth, a smaller sample size due to the specific criteria 314 (participants with all information for the interaction test) limited the power to detect significant interactions for 315 very small effects and can increase selection bias. For the main hypothesis, an effect size of $\mathrm{f}^{2}=0.015$ as found for $316 \mathrm{rs} 3093068 *$ Mediterranean diet still resulted in a power of 0.82 . Finally, this study is rather exploratory as no 317 adjustment for multiple testing was implemented. Since the moderation effect of the Mediterranean diet score was 318 tested thrice (for 3 SNP's), a p-value<0.017 could be used as significance level: even in that case the overall 319 Mediterranean diet score was a significant moderator but the fish component would lose its significance. Indeed, the examination of the subgroups was rather a subhypothesis to detect which subgroups are responsible for the observed overall effect. 


\section{Conclusion}

323

324

325

326

327

328

329

330

331

332

333

334

335

336

337

338

339

340

341

342

343

344

345

346

347

348

This cross-sectional study confirmed that $C R P$ SNPs are related to higher blood CRP concentrations in

European adolescents and also showed the moderating potential of the Mediterranean diet herein. Indeed, greater adherence to the Mediterranean diet and high fish intake were associated with a slight attenuation of the rs3093068 SNP genetic risk towards higher CRP levels. This was not the case for the two other SNPs. We encourage other investigations to reproduce this hypothesis. The results may have practical and clinical importance if causality is confirmed. As most people are far from meeting the basic dietary recommendations, a dietary lifestyle towards a more general Mediterranean diet with a high plant-based intake and sufficient omega-3 would be recommended. By advancing the knowledge of the interaction between genetics and nutrition, better prevention strategies can be developed with nutrition as a cornerstone.

\section{Funding sources}

The HELENA Study was supported by the European Community Sixth RTD Framework Programme (Contract FOODCT-2005-007034). The writing group takes sole responsibility for the content of this article. The European Community is not liable for any use that may be made of the information contained therein.

\section{Research data}

The authors do not have permission to share the entire dataset.

\section{Conflicts of interest}

The authors declare no competing interests.

\section{Credit authorship contribution statement}

Aline Arouca formulated the research question, has analyzed the data and wrote a draft of the paper. Nathalie Michels helped in formulating the research question, analyzing the data and did editing of the first draft. Nathalie Michels and Stefaan De Henauw are co-supervisor and supervisor of Aline Arouca; Luis Moreno was coordinator of the HELENA project. Aline Meirhaeghe and Jean Dallongeville were responsible for the DNA 
349 extraction and genotyping of the SNPs. Gustavo Lourenço performed the statistical analyzes on SNPs. Marcela 350 González-Gross was responsible for blood sampling and collection. Ascensión Marcos was responsible for the 351 inflammatory parameter analyses. Inge Huybrechts developed the Mediterranean diet score. All authors have made 352 substantial contributions to all of the following: (1) data collection, (2) interpretation of data, (3) revising the article 353 critically for important intellectual content, and (4) final approval of the manuscript.

354

355 Acknowledgment

356

The authors acknowledge the Laboratoire d'Analyse Génomique Centre de Ressources Biologiques 357 (LAG-CRB) - BB-0033-00071 Institut Pasteur de Lille, F-59000 Lille, France for managing human samples. 358

359

360

361

362

363 


\section{References}

[1] Strandberg TE, Tilvis RS. C-reactive protein, cardiovascular risk factors, and mortality in a prospective study in the elderly. Arter Thromb Vasc Biol 2000;20:1057-60.

[2] Wang A, Liu J, Li C, Gao J, Li X, Chen S, et al. Cumulative Exposure to High-Sensitivity C-Reactive Protein Predicts the Risk of Cardiovascular Disease. J Am Hear Assoc 2017;6. doi:10.1161/JAHA.117.005610.

[3] Carlson CS, Aldred SF, Lee PK, Tracy RP, Schwartz SM, Rieder M, et al. Polymorphisms within the Creactive protein (CRP) promoter region are associated with plasma CRP levels. Am J Hum Genet 2005;77:64-77. doi:10.1086/431366.

[4] Kushner I, Samols D, Magrey M. A Unifying Biologic Explanation for "High-Sensitivity" C-Reactive Protein and "Low-Grade” Inflammation. Am Coll Rheumatol 2010;62:442-446. doi:10.1002/acr.20052.

[5] Cook DG, Mendall MA, Whincup PH, Carey IM, Ballam L, Morris JE, et al. C-reactive protein concentration in children: relationship to adiposity and other cardiovascular risk factors. Atherosclerosis 2000;149:139-50.

[6] Hage FG, Szalai AJ. C-reactive protein gene polymorphisms, C-reactive protein blood levels, and cardiovascular disease risk. J Am Coll Cardiol 2007;50:1115-22. doi:10.1016/j.jacc.2007.06.012.

[7] Lambert M, Delvin EE, Paradis G, O’Loughlin J, Hanley JA, Levy E. C-reactive protein and features of the metabolic syndrome in a population-based sample of children and adolescents. Clin Chem 2004;50:1762-8. doi:10.1373/clinchem.2004.036418.

[8] Ford ES, Galuska DA, Gillespie C, Will JC, Giles WH, Dietz WH. C-reactive protein and body mass index in children: findings from the Third National Health and Nutrition Examination Survey, 19881994. J Pediatr 2001;138:486-92. doi:10.1067/mpd.2001.112898.

[9] DeBoer MD. Obesity, systemic inflammation, and increased risk for cardiovascular disease and diabetes among adolescents: a need for screening tools to target interventions. Nutrition 2013;29:379-86. doi:10.1016/j.nut.2012.07.003.

[10] Ferrari M, Cuenca-García M, Valtueña J, Moreno LA, Censi L, González-Gross M, et al. Inflammation profile in overweight/obese adolescents in Europe: an analysis in relation to iron status. Eur J Clin Nutr 
2015;69:247-55. doi:10.1038/ejen.2014.154.

[11] Lee CC, You NC, Song Y, Hsu YH, Manson J, Nathan L, et al. Relation of genetic variation in the gene coding for C-reactive protein with its plasma protein concentrations: findings from the Women's Health Initiative Observational Cohort. Clin Chem 2009;55:351-60. doi:10.1373/clinchem.2008.117176.

[12] Kolz M, Koenig W, Müller M, Andreani M, Greven S, Illig T, et al. DNA variants, plasma levels and variability of C-reactive protein in myocardial infarction survivors: results from the AIRGENE study. Eur Hear J 2008;29:1250-8. doi:10.1093/eurheartj/ehm442.

[13] Halder I, Marsland AL, Cheong J, Muldoon MF, Ferrell RE, Manuck SB. Polymorphisms in the CRP gene moderate an association between depressive symptoms and circulating levels of C-reactive protein. Brain Behav Immun 2010;24:160-7. doi:10.1016/j.bbi.2009.09.014.

[14] Gaysina D, Pierce M, Richards M, Hotopf M, Kuh D, Hardy R. Association between adolescent emotional problems and metabolic syndrome: the modifying effect of C-reactive protein gene (CRP) polymorphisms. Brain Behav Immun 2011;25:750-8. doi:10.1016/j.bbi.2011.01.019.

[15] Curti ML, Jacob P, Borges MC, Rogero MM, Ferreira SR. Studies of gene variants related to inflammation, oxidative stress, dyslipidemia, and obesity: implications for a nutrigenetic approach. J Obes 2011;2011:497401. doi:10.1155/2011/497401.

[16] Kaput J, Ordovas JM, Ferguson L, van Ommen B, Rodriguez RL, Allen L, et al. The case for strategic international alliances to harness nutritional genomics for public and personal health. Br J Nutr 2005;94:623-32.

[17] Muñoz A, Costa M. Nutritionally mediated oxidative stress and inflammation. Oxid Med Cell Longev 2013;2013:610950. doi:10.1155/2013/610950.

[18] Root MM, McGinn MC, Nieman DC, Henson DA, Heinz SA, Shanely RA, et al. Combined fruit and vegetable intake is correlated with improved inflammatory and oxidant status from a cross-sectional study in a community setting. Nutrients 2012;4:29-41. doi:10.3390/nu4010029.

[19] O’Keefe JH, Gheewala NM, O'Keefe JO. Dietary strategies for improving post-prandial glucose, lipids, inflammation, and cardiovascular health. J Am Coll Cardiol 2008;51:249-55. doi:10.1016/j.jacc.2007.10.016. 
[20] Calder PC, Ahluwalia N, Brouns F, Buetler T, Clement K, Cunningham K, et al. Dietary factors and lowgrade inflammation in relation to overweight and obesity. Br J Nutr 2011;106 Suppl:S5-78. doi:10.1017/S0007114511005460.

[21] Arouca A, Michels N, Moreno LA, González-Gil EM, Marcos A, Gómez S, et al. Associations between a Mediterranean diet pattern and inflammatory biomarkers in European adolescents. Eur J Nutr 2018;57. doi:10.1007/s00394-017-1457-4.

[22] Moreno LA, De Henauw S, González-Gross M, Kersting M, Molnár D, Gottrand F, et al. Design and implementation of the Healthy Lifestyle in Europe by Nutrition in Adolescence Cross-Sectional Study. Int J Obes 2008;32 Suppl 5:S4-11. doi:10.1038/ijo.2008.177.

[23] Diethelm K, Huybrechts I, Moreno L, De Henauw S, Manios Y, Beghin L, et al. Nutrient intake of European adolescents: results of the HELENA (Healthy Lifestyle in Europe by Nutrition in Adolescence) Study. Public Heal Nutr 2014;17:486-97. doi:10.1017/S1368980013000463.

[24] Vereecken CA, Covents M, Matthys C, Maes L. Young adolescents' nutrition assessment on computer (YANA-C). Eur J Clin Nutr 2005;59:658-67. doi:10.1038/sj.ejcn.1602124.

[25] Trichopoulou A, Kouris-Blazos A, Wahlqvist ML, Gnardellis C, Lagiou P, Polychronopoulos E, et al. Diet and overall survival in elderly people. BMJ 1995;311:1457-60.

[26] Trichopoulou A, Costacou T, Bamia C, Trichopoulos D. Adherence to a Mediterranean diet and survival in a Greek population. N Engl J Med 2003;348:2599-608. doi:10.1056/NEJMoa025039.

[27] Trichopoulou A. Traditional Mediterranean diet and longevity in the elderly: a review. Public Heal Nutr 2004;7:943-7.

[28] Nagy E, Vicente-Rodriguez G, Manios Y, Béghin L, Iliescu C, Censi L, et al. Harmonization process and reliability assessment of anthropometric measurements in a multicenter study in adolescents. Int J Obes 2008;32 Suppl 5:S58-65. doi:10.1038/ijo.2008.184.

[29] Cole TJ, Bellizzi MC, Flegal KM, Dietz WH. Establishing a standard definition for child overweight and obesity worldwide: international survey. BMJ 2000;320:1240-3. doi: 10.1136/bmj.320.7244.1240.

[30] Jiménez Pavón D, Ortega FP, Ruiz JR, España Romero V, García Artero E, Moliner Urdiales D, et al. Socioeconomic status influences physical fitness in European adolescents independently of body fat and 
physical activity: the HELENA study. Nutr Hosp 2010;25:311-6.

[31] González-Gross M, Breidenassel C, Gómez-Martínez S, Ferrari M, Béghin L, Spinneker A, et al. Sampling and processing of fresh blood samples within a European multicenter nutritional study: evaluation of biomarker stability during transport and storage. Int J Obes 2008;32 Suppl 5:S66-75. doi:10.1038/ijo.2008.185.

[32] Hayes AF, Rockwood NJ. Regression-based statistical mediation and moderation analysis in clinical research: Observations, recommendations, and implementation. Behav Res Ther 2016. doi:10.1016/j.brat.2016.11.001.

[33] Hayes AF. Introduction to mediation, moderation, and conditional process analysis: A regression-based approach. Guilford Publications; 2013.

[34] Nimptsch K, Aleksandrova K, Boeing H, Janke J, Lee YA, Jenab M, et al. Association of CRP genetic variants with blood concentrations of C-reactive protein and colorectal cancer risk. Int J Cancer 2015;136:1181-92. doi:10.1002/ijc.29086.

[35] Esposito K, Giugliano D. Diet and inflammation: a link to metabolic and cardiovascular diseases. Eur Hear J 2006;27:15-20. doi:10.1093/eurheartj/ehi605.

[36] Esposito K, Marfella R, Ciotola M, Di Palo C, Giugliano F, Giugliano G, et al. Effect of a mediterraneanstyle diet on endothelial dysfunction and markers of vascular inflammation in the metabolic syndrome: a randomized trial. JAMA 2004;292:1440-6. doi:10.1001/jama.292.12.1440.

[37] Wall R, Ross RP, Fitzgerald GF, Stanton C. Fatty acids from fish: the anti-inflammatory potential of long-chain omega-3 fatty acids. Nutr Rev 2010;68:280-9. doi:10.1111/j.1753-4887.2010.00287.x.

[38] Sureda A, Bibiloni MDM, Julibert A, Bouzas C, Argelich E, Llompart I, et al. Adherence to the Mediterranean Diet and Inflammatory Markers. Nutrients 2018;10:62. doi:10.3390/nu10010062.

[39] Ajala O, English P, Pinkney J. Systematic review and meta-analysis of different dietary approaches to the management of type 2 diabetes. Am J Clin Nutr 2013;97:505-16. doi:10.3945/ajcn.112.042457.

[40] Esposito K, Maiorino MI, Ceriello A, Giugliano D. Prevention and control of type 2 diabetes by Mediterranean diet: a systematic review. Diabetes Res Clin Pr 2010;89:97-102. doi:10.1016/j.diabres.2010.04.019. 
[41] Llorente-Cortés V, Estruch R, Mena MP, Ros E, González MAM, Fitó M, et al. Effect of Mediterranean diet on the expression of pro-atherogenic genes in a population at high cardiovascular risk. Atherosclerosis 2010;208:442-50. doi:10.1016/j.atherosclerosis.2009.08.004.

[42] Crider KS, Yang TP, Berry RJ, Bailey LB. Folate and DNA methylation: a review of molecular mechanisms and the evidence for folate's role. Adv Nutr 2012;3:21-38. doi:10.3945/an.111.000992.

[43] Vaitla B, Collar D, Smith MR, Myers SS, Rice BL, Golden CD. Predicting nutrient content of ray-finned fishes using phylogenetic information. Nat Commun 2018;9:3742. doi:10.1038/s41467-018-06199-w.

[44] Kris-Etherton PM, Harris WS, Appel LJ, Committee N. Fish consumption, fish oil, omega-3 fatty acids, and cardiovascular disease. Arter Thromb Vasc Biol 2003;23:e20-30.

[45] Parnell LD, Blokker BA, Dashti HS, Nesbeth PD, Cooper BE, Ma Y, et al. CardioGxE, a catalog of geneenvironment interactions for cardiometabolic traits. BioData Min 2014;7:21. doi:10.1186/1756-0381-721.

[46] Raqib R, Cravioto A. Nutrition, immunology, and genetics: future perspectives. Nutr Rev 2009;67 Suppl 2:S227-36. doi:10.1111/j.1753-4887.2009.00244.x.

[47] Grimble RF, Howell WM, O’Reilly G, Turner SJ, Markovic O, Hirrell S, et al. The ability of fish oil to suppress tumor necrosis factor alpha production by peripheral blood mononuclear cells in healthy men is associated with polymorphisms in genes that influence tumor necrosis factor alpha production. Am J Clin Nutr 2002;76:454-9. doi:10.1093/ajen/76.2.454.

[48] Nienaber-Rousseau C, Swanepoel B, Dolman RC, Pieters M, Conradie KR, Towers GW. Interactions between C-reactive protein genotypes with markers of nutritional status in relation to inflammation. Nutrients 2014;6:5034-50. doi:10.3390/nu6115034.

[49] Sarría A, García-Llop LA, Moreno LA, Fleta J, Morellón MP, Bueno M. Skinfold thickness measurements are better predictors of body fat percentage than body mass index in male Spanish children and adolescents. Eur J Clin Nutr 1998;52:573-6. 


\section{Figure and Tables legends}

Figure 1. The $C R P$ gene region (chromosome 1q21-q23) and the locations of the three SNPs tested in our study.

Figure 2. Sampling procedure scheme.

Figure 3. Moderation by diet on the relation between CRP rs3093068 SNP and CRP blood concentrations.

(A) Moderating effect of the adherence to the Mediterranean diet on CRP levels among adolescents with and without the CC genotype of rs3093068.

(B) Moderating effect of the fish subgroup intake on CRP blood concentrations among adolescents with and without the CC genotype of rs3093068.

Table 1. Characteristics of the study sample.

Table 2. Associations between the allele frequencies of the investigated $C R P$ genotypes (rs3093068, rs1205 and rs1130864) and CRP blood concentrations in the HELENA study.

Table 3. Moderating effects of diet on the relation between CRP gene SNPs (rs3093068, rs1205, rs1130864) and CRP blood concentrations in 562 adolescents from the HELENA study. 